\title{
The Effects of Clinorotation on the Host Plant, Medicago truncatula, and Its Microbial Symbionts
}

OPEN ACCESS

Edited by:

Jack Van Loon,

VU University, Netherlands

Reviewed by:

Zurab Silagadze,

Budker Institute of Nuclear Physics,

Russia

Ruediger Hampp,

University of Tuebingen, Germany

Hideyuki Takahashi,

Tohoku University, Japan

*Correspondence:

John Z. Kiss

jzkiss@olemiss.edu

Specialty section:

This article was submitted to

Cosmology,

a section of the journal

Frontiers in Astronomy and Space

Sciences

Received: 29 September 2015 Accepted: 08 February 2016

Published: 26 February 2016

Citation:

Dauzart AJC, Vandenbrink JP and

Kiss JZ (2016) The Effects of

Clinorotation on the Host Plant,

Medicago truncatula, and Its Microbial

Symbionts.

Front. Astron. Space Sci. 3:3

doi: 10.3389/fspas.2016.00003

\author{
Ariel J. C. Dauzart, Joshua P. Vandenbrink and John Z. Kiss * \\ Department of Biology, Graduate School, University of Mississippi, University, MS, USA
}

Understanding the outcome of the plant-microbe symbiosis in reduced or altered is vital to developing life support systems for long-distance space travel and colonization of other planets. Thus, the aim of this research was to understand mutualistic relationships between plants and endophytic microbes under the influence of altered gravity. This project utilized the model tripartite relationship among Medicago truncatula-Sinorhizobium meliloti-Rhizophagus irregularis. Plants were inoculated with rhizobial bacteria (S. meliloti), arbuscular mycorrhizal fungi $(R$. irregularis), or both microbes, and placed on a rotating clinostat. Vertical and horizontal static controls were also performed. Clinorotation significantly reduced $M$. truncatula dry mass and fresh mass compared to the static controls. The addition of rhizobia treatments under clinorotation also altered total root length and root-to-shoot fresh mass ratio. Nodule size decreased under rhizobia + clinorotation treatment, and nodule density was significantly decreased compared to the vertical treatment. However, inoculation with arbuscular mycorrhizal fungi was shown to increase biomass accumulation and nodule size. Thus, clinorotation significantly affected $M$. truncatula and its symbiotic relationships with S. meliloti and R. irregularis. In the long term, the results observed in this clinostat study on the changes of plant-microbe mutualism need to be investigated in spaceflight experiments. Thus, careful consideration of the symbiotic microbes of plants should be included in the design of bioregenerative life support systems needed for space travel.

Keywords: Clinorotation, Medicago truncatula, nodulation, plant biology, plant symbiosis, Rhizophagus irregularis, Sinorhizobium meliloti, space biology

\section{INTRODUCTION}

To achieve the goal of long-distance space travel, researchers need to first develop a biological selfsustained life support system (Ferl et al., 2002). Plants constitute the cornerstone of this system as they have the ability to recycle waste water for drinking and carbon dioxide into breathable oxygen (National Research Council, 2011). The National Research Council also states a need for "basic plant and microbial research to define how these organisms sense and respond to the varied environments presented in space." Using the legume Medicago truncatula as a model for plant space biology research, new information can be obtained on the effects of reduced gravity on a valuable crop species and two of its associated microbial symbionts, rhizobial bacteria, and arbuscular mycorrhizal fungi (AMF). To date, the relationship among legumes, rhizobia and AMF has not previously been studied under reduced gravity. However, studying these symbioses under an altered gravity should provide insight into the role that gravity plays in this symbiotic tripartite relationship on Earth and beyond. 
M. truncatula is a plant that is a fast growing, herbaceous legume, and it forms symbiotic relationships with both rhizobia and AMF. Interest in M. truncatula is also due to the agriculturally significant close relative, Medicago sativa, or alfalfa (Kouchi et al., 2010). M. truncatula is a forage crop but is used as a model legume due to its relatively small diploid genome $(2 n=16)$ and autogamous reproduction, whereas alfalfa contains a larger tetraploid genome and obligate outbreeding reproduction (Connor et al., 2011). More importantly, M. truncatula forms symbiotic relationships with multiple arbuscular mycorrhizal species, including Rhizophagus irregularis (formerly Glomus intraradices; Hogekamp and Kuster, 2013) and with rhizobial bacteria, including Sinorhizobium meliloti.

Research to date suggests that plants can survive and produce a viable food source on the ISS; however, these plants may be stressed growing in microgravity conditions and may not be reaching their full potential as a food source (Colla et al., 2007; Wolverton and Kiss, 2009; Vandenbrink and Kiss, 2016). On Earth, plants have microbial symbionts that are beneficial under stressful conditions such as increased salinity, drought, and heavy metal toxicity (Nadeem et al., 2014). However, altered gravity is a stressor that plants have not evolved to handle. Thus, to date, there is a lack of published information on how colonization of symbiotic microbes such as AMF and rhizobia changes under altered gravity. We cannot be sure that a symbiotic relationship will remain unchanged because there are changes within a plant's growth and physiological responses under an altered gravity.

AMF colonize between 80 and $90 \%$ of plant species, including our most valuable food crops (Smith and Read, 2008). Legumes colonized by AMF benefit from a mutualistic relationship with the fungi, leading to an increase in phosphorus $(\mathrm{P})$ and nitrogen (N) uptake by the plant (Wang and Dong, 2011; Larimer and Bever, 2014). Legumes colonized with AMF can produce more protein rich foods and tolerate abiotic stressors more vigorously as a result of an increase in nutrient availability (Vazquez et al., 2001; Ashrafi et al., 2014). Rhizobia colonize $88 \%$ of legumes and fix atmospheric nitrogen into a useable form for plants to assimilate into proteins and nucleic acids (Graham and Vance, 2003). Legumes benefit from forming a mutualistic tripartite symbiosis with both rhizobia and AMF through nutrient uptake greater than the individual microbe alone could provide the host (Larimer et al., 2010). The dual colonized host also is more resistant to pathogens and abiotic stressors such as drought, increased soil salinity and metal toxicity (Nadeem et al., 2014). Thus, plants grown with symbiotic microbes in an Advanced Life Support (ALS) system would be more efficient at assimilating the vital elements, $\mathrm{P}$ and $\mathrm{N}$, within foods essential to astronauts.

Ground-based methods of altering gravity provide a practical method to studying organisms under such conditions (Herranz et al., 2013). There are many instruments that can simulate the effects of an altered gravity vector, and these include diamagnetic levitation (Qian et al., 2013), random positioning machines (RPMs; Kraft et al., 2000; Herranz et al., 2013) and 2-dimensional (2D) clinostats, the latter which we employ here. This device will rotate organisms at a programmed speed on a single axis. In addition, these instruments can provide valuable, preliminary information as to how organisms will react to microgravity conditions (Kraft et al., 2000). Many studies with plants under altered gravity that have implemented clinostats have found similar results (depending on the parameter) to microgravity conditions in space (Herranz et al., 2013). Gravity-sensing plant cells in the columella contain dense starch granules that cannot settle under clinorotation due to the constant alteration of the gravity vector. Because these amyloplasts within the columella do not settle, the plant is unable to detect the direction of gravity (Kiss, 2000). In many cases, clinostats and RPMs provide similar results in experiments in true microgravity. For instance, Matia et al. (2010) studied Arabidopsis meristematic growth on an RPM and true microgravity on the ISS. The two experimental groups produced similar results that were significantly different from the $1 \mathrm{~g}$ controls on the ISS and Earth. This research team concluded that, for the parameters measured, an RPM was comparable to spaceflight experiments on Arabidopsis meristematic tissue. In addition, Kraft et al. (2000) used an RPM to study Arabidopsis plastid (amyloplast) position in columella cells. The results indicated that the location of the plastids within the cell on the RPM was similar to those from a true microgravity study in spaceflight.

In the future, humans that travel long distances beyond the Earth's atmosphere for extended periods of time will be reliant on an ALS system. This system will be a small scale replication of a quintessential ecosystem pieced together from organisms found in ecosystems on Earth. At the base of any cyclical system that humans rely on for survival, plants will serve as cornerstone organisms. Although plants can produce food, recycle wastes into water and oxygen autonomously, host plants are more efficient with microbial symbionts assisting in nutrient uptake. Beneficial plant-microbe symbioses have the potential for many advantages in a self-sustainable system, but this relationship has not yet been studied in an agriculturally important crop plant in a groundbased, gravity-vector-altering apparatus nor has it been studied on the ISS. Thus, we report on the M. truncatula-S. meliloti-R. irregularis symbiotic model system in order to identify the effects of altered gravity on the plant-microbe mutualistic symbiosis. The major questions addressed in this research are: (1) does $M$. truncatula growth change under altered gravity; (2) does altered gravity affect the tripartite symbiosis among $M$. truncatula-S. meliloti-R. irregularis?

\section{MATERIALS AND METHODS}

M. truncatula seeds (accession A17) were scarified using 98\% (v/v) sulfuric acid for $10 \mathrm{~min}$ followed by a rinse with sterile water. Next, seeds were surface sterilized in $30 \%(\mathrm{v} / \mathrm{v})$ bleach solution for $10 \mathrm{~min}$ followed by a second rinse with water. Scarified seeds were shaken for $4 \mathrm{~h}$ in sterile water. Wet seeds were placed in a $100 \mathrm{~mm}$ sterile Petri dish, turned over so the seeds adhered to the top of the Petri dish, and then were left to germinate overnight. After $24 \mathrm{~h}$, the seedlings were planted in autoclaved Metro Mix 366 soil (Sungro Horticulture, Massachusetts, USA). Seedlings were grown in a growth chamber for 2 weeks $\left(18-22^{\circ} \mathrm{C}, 16 \mathrm{~h}\right.$ light, $8 \mathrm{~h}$ dark) before transplanting to cone-tainers (\#SC10R, Stuewe and Sons, Oregon, USA). 
Kanamycin resistant S. meliloti 1021 was incubated in TY/Ca ${ }^{+}$ broth at $30^{\circ} \mathrm{C}$ for $46-48 \mathrm{~h}$ (Journet et al., 2006). At this point, the rhizobia are in late logarithmic to early stationary stage. The absorbance reading at $600 \mathrm{~nm}$ was between 0.9 and $1.0 \mathrm{OD}$ as assayed with a spectrophotometer. The culture was then rinsed with sterile water three times to remove TY/ $\mathrm{Ca}^{+}$broth, and then re-suspended in sterile water. The rinsed culture's absorbance was measured again at $600 \mathrm{~nm}$ and ranged between 0.8 and 0.9 OD.

$R$. irregularis was grown in sterile conditions in $\mathrm{M}$ media (Bécard and Fortin, 1988) on a split plate. One side (1) of the plate had sucrose added to the media for mutated carrot root to grow on, and the other (side 2) was without sucrose. The AMF was initially placed on side 1 with the carrot root but over time the hyphae spread to side 2 . Spores were harvested from the gel matrix by blending in a Hamilton Beach Single Server Blender (item\# 1568444, Walmart, Arkansas, USA) with $10 \mathrm{mM}$ sodium citrate. The mixture was blended for five, $5 \mathrm{~s}$ intervals. The solution was filtered through $250 \mu \mathrm{m}$ mesh cloth to remove carrot root debris. Only spores and liquid passed through this mesh. To then isolate the spores, we filtered the solution through a $50 \mu \mathrm{m}$ mesh. Spores remained on the mesh and were rinsed with sterile water into a Falcon tube. To quantify the amount of spores collected, $25 \mu \mathrm{L}$ of spore solution was placed onto a microscope slide and examined under a dissecting microscope. The spores from each $5 \mu \mathrm{L}$ drop were counted then averaged. From this average, the concentration of the spore solution was extrapolated.

Each cone-tainer was layered from the bottom of the conetainer to the top as follows: a foam plug (\#98140-960, VWR, Georgia, USA), Profile ${ }^{\circledR}$ Greens Grade porous ceramic as the lowermost layer followed by a $2: 1$ sand to pebble mixture $(125 \mathrm{ml})$, a centimeter of packed sand, and the 2 weeks old seedling on top of the sand layer. The total volume of the conetainer was $164 \mathrm{ml}$. A 1/4 inch polyethylene tube (\#HSVEB20, Watts, Home Depot, USA) was placed into the soil to allow efficient watering of rotating plants via syringe (\#301604, Becton Dickenson Disposable Syringe). The roots of an individual seedling were inoculated with $\sim 800$ spores of $R$. irregularis, $8-9 \times 108$ cells of $S$. meliloti, or both, and subsequently covered with a thin layer of sand $(5-10 \mathrm{ml})$. The cone-tainer was capped by an Erlenmeyer flask foam plug (\#98140-960, VWR, Georgia, USA). Inoculated and non-inoculated (microbial control) 14day-old plants are rotated for 28 days on a $1 \mathrm{rpm}$ rotating clinostat (Figure 1A). We also inoculated two sets of control plants with the same concentrations of spores and bacteria, and these gravitational control plants were place in a horizontal or vertical position for 28 days (Figure 1B). In addition, noninoculated (microbial control) and non-rotated (gravitational control) plants were set in a vertical and horizontal position as well. A fifth run that only tested AMF and microbial control plants was performed. In total, 186 plants were analyzed for statistical differences.

During each experiment, plants were grown under a $16 \mathrm{~h}$ day/8 h night cycle at $\sim 23^{\circ} \mathrm{C}$. The plants were given $10 \mathrm{ml}$ of distilled water via syringe twice a week through a watering tube. In addition, Hoagland's nutrient solution was diluted to $1 / 8$ concentration and delivered in the same manner as water once a

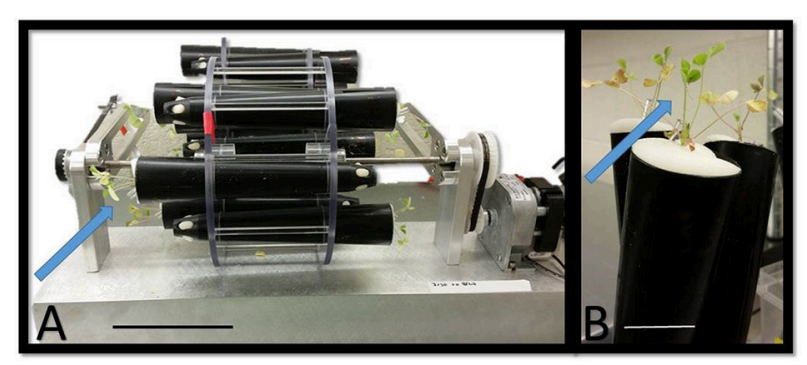

FIGURE 1 | (A) Image of the two-dimensional clinostat used in these studies. (B) Vertical gravitational controls of seedlings transplanted into cone-tainers. Arrows indicate newly transplanted 14-days-old seedlings. (A) Scale bar = $10.5 \mathrm{~cm}$. (B) Scale bar $=3.0 \mathrm{~cm}$

week. After 28 days post inoculation (dpi), plants were removed from the rotating clinostat. Stem length, total root length, and total biomass were measured as well as nodule number and size. Root tissue was cleaned of soil, photographed, and placed into $50 \%(\mathrm{v} / \mathrm{v})$ ethanol. Aerial tissue was dried at $60^{\circ} \mathrm{C}$ in an oven for $48 \mathrm{~h}$. The total root length was measured using the images taken at the end of each experiment in program GIA Roots (Galkovskyi et al., 2012). The same images were used to measure nodule size using the program Image (Rasband, 2014).

Data were analyzed using two- and three-way analysis of variance (ANOVA) and post hoc tests in the program R (version 3.2.1) with the "car" and "multcomp" packages. The response variables tested in the three-way ANOVA models were dry mass of aerial tissue, change in total fresh biomass after $28 \mathrm{dpi}$, total root length, and root:shoot fresh mass ratio. Each three-way ANOVA model was run using the two microbial (+/ - AMF and +/- Rhizobia) predictor variables and gravitational predictor variables (GrT: vertical, horizontal, and clinorotated), as well as with the interactions among those three variables. Two-way ANOVA tests were performed for the response variables-nodule size, nodule density, and AMF percent colonization. For analysis of nodule size and nodule density, we utilized plants that were inoculated with rhizobia. This data subset was analyzed with twoway ANOVA models containing the predictor variables-AMF, $\mathrm{GrT}$, and the AMF $\times$ GrT interaction. For analyses of nodule size and nodule density, we analyzed only plants that were inoculated with AMF and this subset was used for a two-way ANOVA model that included the predictor variables rhizobia, GrT, and the rhizobia $\times$ GrT interaction. After the ANOVA tests were performed and significance among treatments was found, means were compared using Tukey's Honestly Significant Difference test $(p<0.05)$. The relationship between dry mass of above-ground tissue and nodule size was analyzed in Pearson's Correlation Test.

\section{RESULTS}

The goal of this study was to use clinorotation to provide altered gravity conditions in order to analyze any potential changes in the symbiotic relationships between the host plant and its associated symbiotic fungi and bacteria. When M. truncatula was grown under altered gravity on a clinostat for 28 days after being 


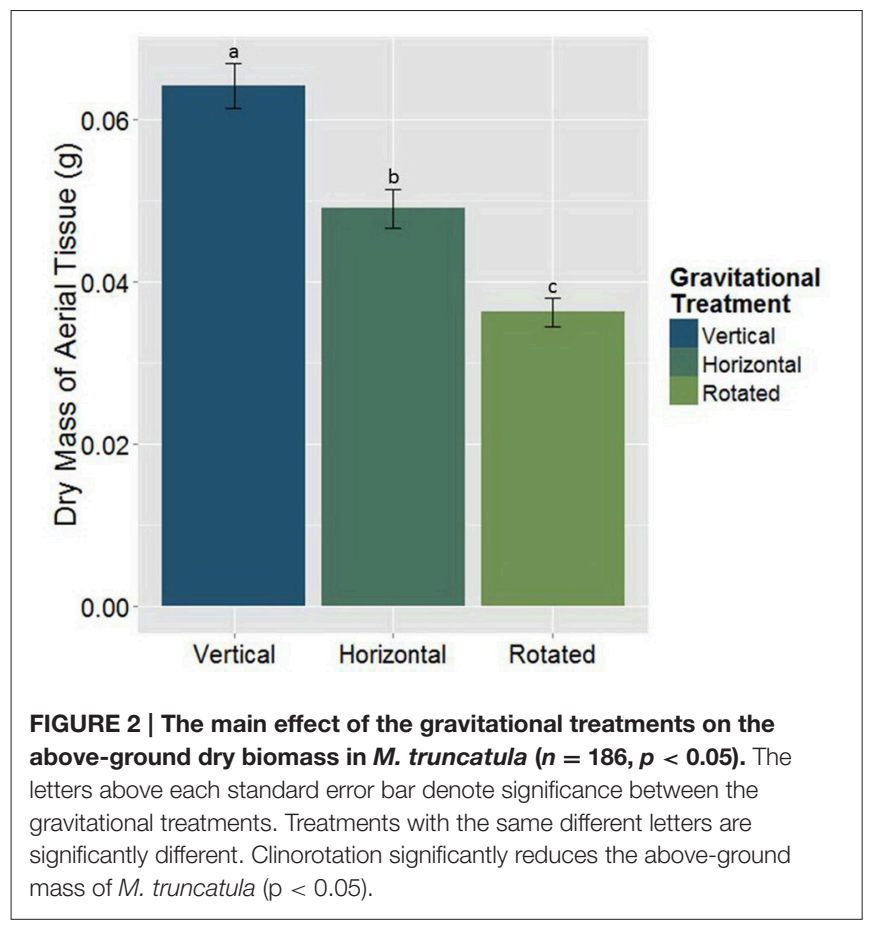

inoculated with AMF, rhizobia, or both symbionts, it exhibited significant changes in distribution of above- and below-ground biomass. Thus, clinorotation produces a significant reduction of dry aerial tissue relative to the vertical control and the horizontal control, which also differed from each other (Figure 2, Table 1). At no time did any plants exhibit signs of drought response in any of the three orientation conditions, suggesting adequate access to water in these experiments. Interestingly, microbial treatments appear to have no significant effect $(p<0.05)$ on dry mass of aerial tissue under altered gravity.

Total fresh biomass accumulation after 28 dpi was influenced by significant main effects of AMF, rhizobia, and gravitational treatment (GrT; Table 2). Plants inoculated with AMF gained significantly more fresh mass compared to plants without AMF (Figure 3A, $p<0.05$ ). The inoculation with rhizobia had the opposite effect on plants. Thus, nodulated plants gained significantly less fresh mass than groups without nodules (Figure 3B, $p<0.05$ ). Clinorotation reduced the fresh mass accumulated over 28 days, and the vertical treatment had the largest fresh mass gain (Figure 3C).

The total root length was measured to assess below-ground morphological changes. There was a significant interaction effect between the rhizobial and gravitational treatments (Figure 4, Table 3) resulting in total root length being much more reduced in nodulated plants compared to non-nodulated plants $(p<0.05$, Figure 4). Rhizobia-treated plants in the vertical (Figure 5A) and horizontal (Figure 5B) control groups developed significantly greater roots lengths than the rhizobia + clinorotated group respectively (Figure 5C).

The root:shoot fresh biomass ratio was measured as an indication of the plants' allocation of resources within the plants. There was a significant interaction between the rhizobia
TABLE 1 | Three-way ANOVA results for dry mass of the above-ground tissue in microbial, gravitational treatments (GrT), and microbial $\times$ gravitational treatment groups.

Three-way ANOVA of dry mass in aerial tissue

\begin{tabular}{lccc}
\hline Treatment & $\boldsymbol{d f}$ & $\boldsymbol{F}$-value & $\boldsymbol{p}$-value \\
\hline AMF & 1 & 3.54 & 0.061 \\
Rhizobia & 1 & 0.75 & 0.387 \\
${ }^{*}$ GrT & 2 & 6.04 & 0.003 \\
AMF $\times$ Rhizobia & 1 & 1.87 & 0.172 \\
AMF $\times$ GrT & 2 & 0.39 & 0.677 \\
Rhizobia $\times$ GrT & 2 & 0.80 & 0.451 \\
AMFx Rhiz $\times$ GrT & 2 & 0.08 & 0.920
\end{tabular}

Significant differences are indicated by an asterisk $(p<0.05)$.

TABLE 2 | Three-way ANOVA results indicating the significance in the change in fresh biomass of $M$. truncatula $28 \mathrm{dpi}$ and gravitational treatment (GrT).

Three-way ANOVA of change in fresh mass

\begin{tabular}{lccc}
\hline Treatment & $\boldsymbol{d} \boldsymbol{f}$ & $\boldsymbol{F}$-value & $\boldsymbol{p}$-value \\
\hline${ }^{*} \mathrm{AMF}$ & 1 & 8.38 & $4.29 \times 10^{-3}$ \\
${ }^{*}$ Rhizobia & 1 & 32.34 & $5.38 \times 10^{-8}$ \\
${ }^{*} \mathrm{GrT}$ & 2 & 43.78 & $3.96 \times 10^{-6}$ \\
AMF $\times$ Rhizobia & 1 & 2.23 & 0.137 \\
AMF $\times$ GrT & 2 & 1.21 & 0.300 \\
Rhizobia $\times$ GrT & 2 & 1.33 & 0.266 \\
AMFx Rhiz $\times$ GrT & 2 & 0.04 & 0.961 \\
\hline
\end{tabular}

AM fungi and rhizobia treatments and GrT created a main effect in the host plant's ability to accumulate fresh biomass. Significant differences are indicated by asterisks $(p<0.05)$.

TABLE 3 | Three-way ANOVA results indicating the significance of total root length with a combination of microbial treatments, gravitational treatments (GrT) and a combination of both.

Three-way ANOVA results of total root length

\begin{tabular}{lccc}
\hline Treatment & $\boldsymbol{d} \boldsymbol{f}$ & $\boldsymbol{F}$-value & $\boldsymbol{p}$-value \\
\hline AMF & 1 & 2.21 & 0.139 \\
Rhizobia & 1 & 0.02 & 0.896 \\
${ }^{*}$ GrT & 2 & 18.17 & $6.84 \times 10^{-8}$ \\
AMF $\times$ Rhizobia & 1 & 0.52 & 0.471 \\
AMF $\times$ GrT & 2 & 1.43 & 0.243 \\
*Rhizobia $\times$ GrT & 2 & 6.22 & $2.46 \times 10^{-3}$ \\
AMFx Rhiz $\times$ GrT & 2 & 0.07 & 0.934 \\
\hline
\end{tabular}

Significant differences are indicated by asterisks $(p<0.05)$.

inoculum and GrT on the root:shoot ratio (Figure 6, Table 4). Non-nodulated plants gained more below-ground biomass compared to rhizobia-inoculated plants, as indicated by the overall higher root:shoot ratio., but differences among the gravitational treatments were much more dramatic in the nonnodulated plants compared to the nodulated plants. 


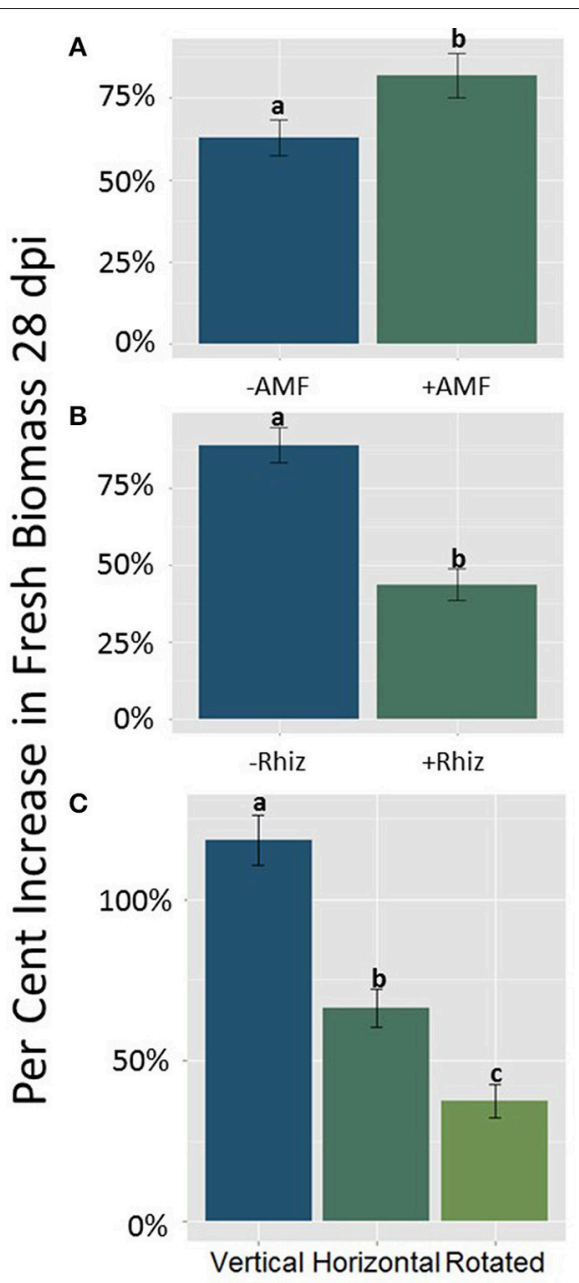

FIGURE 3 | (A-C) The main effects of AMF (A), rhizobia (B), and gravitational treatment $(\mathbf{C})$ on the difference in fresh mass of seedlings 28 days post inoculation (dpi) in the host, $M$. truncatula. (A) AMF treated plants from all three orientations $(n=94)$ gained significantly more fresh mass compared to plants not treated with AMF $(p<0.05)$. (B) Rhizobia treated plants from all three orientations ( $n=69$ ) gained significantly less fresh mass compared to plants not treated with rhizobia $(p<0.05)$. (C) The clinorotated plants $(n=66)$ gained less fresh mass compared to vertically and horizontally grown plants $(p<0.05$; $n=58$ and $n=62$, respectively). The letters above each standard error bar denote significance between treatments.

Alterations in the gravity treatment interacted with AMF inoculation to influence nodule size (Figure 7, Table 5) and had a significant main effect on nodule density (Figure 8, Table 6). Plants inoculated with AMF did not have significantly different nodule sizes among gravitational treatments, whereas plants not inoculated with AMF exhibited negative effects of clinorotation on nodule size. Rhizobia-inoculated plants produced a reduced number of nodules per centimeter under clinorotation compared to the vertical control (Figure 8, $p<0.05$ ).

In addition, gravity did not affect the percent colonization of $R$. irregularis and neither did the addition of rhizobia in the inoculum (data not shown). The mean number \pm SE of nodules for vertical plants inoculated with rhizobia only was $53.5 \pm 5.6$

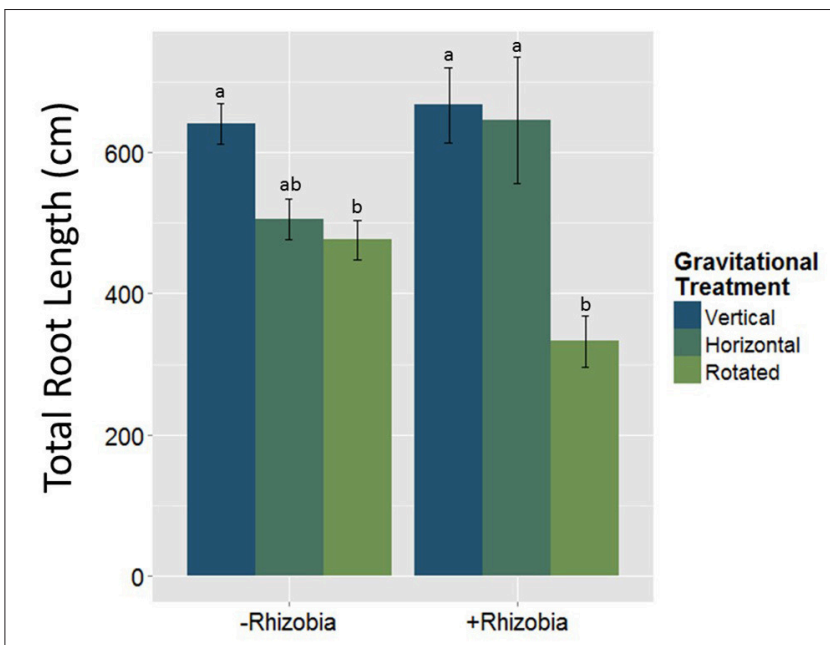

FIGURE 4 | The interaction between rhizobia $\times$ gravitational treatment in total root length. Letters indicate significance among nodulated treated plant (+Rhizobia) and non-nodulated plants (-Rhizobia). Plants treated with rhizobia $(n=69)$ had significantly less total root length $(p<0.05)$. Total root length is significantly reduced in clinorotated plants inoculated with rhizobia ( $n=30$ ) compared to the vertically and horizontally grown plants $(p<0.05$; $n=19$ and $n=20$ ). The letters above each standard error bar denote significance between rhizobia treatments.

TABLE 4 | Three-way ANOVA results of root:shoot fresh mass of $M$. truncatula $28 \mathrm{dpi}$ after microbial inoculation and gravitational treatments (GrT).

Three-way ANOVA results of root:shoot fresh mass

\begin{tabular}{lccc}
\hline Treatment & $\boldsymbol{d} \boldsymbol{f}$ & $\boldsymbol{F}$-value & $\boldsymbol{p}$-value \\
\hline AMF & 1 & 0.40 & 0.529 \\
${ }^{*}$ Rhizobia & 1 & 154.61 & $2.20 \times 10^{-16}$ \\
${ }^{*}$ GrT & 2 & 37.45 & $2.97 \times 10^{-14}$ \\
AMF $\times$ Rhizobia & 1 & 1.20 & 0.274 \\
AMF $\times$ GrT & 2 & 2.05 & 0.132 \\
${ }^{*}$ Rhizobia $\times$ GrT & 2 & 4.29 & 0.015 \\
AMFx Rhiz $\times$ GrT & 2 & 0.14 & 0.872 \\
\hline
\end{tabular}

Significant differences are indicated by asterisks $(p<0.05)$.

TABLE 5 | Two-way ANOVA results of nodule size in rhizobia and AMF +rhizobia treated host plants under gravitational treatments (GrT).

Two-way ANOVA results of nodule size

\begin{tabular}{llll}
\hline Treatment & $\boldsymbol{d f}$ & $\boldsymbol{F}$-value & $\boldsymbol{p}$-value \\
\hline${ }^{*} \mathrm{AMF}$ & 1 & 5.39 & 0.024 \\
$\mathrm{GrT}$ & 2 & 1.93 & 0.153 \\
${ }^{*} \mathrm{AMF} \times \mathrm{GrT}$ & 2 & 3.41 & 0.039
\end{tabular}

Significant differences are indicated by an asterisk $(p<0.05)$.

$(N=10)$ and $73.9 \pm 4.1(N=10)$ for vertical plants inoculated with both microbes. For horizontal plants inoculated, the mean \pm SE nodule count was $45 \pm 4.2(N=10)$ for plants inoculated 

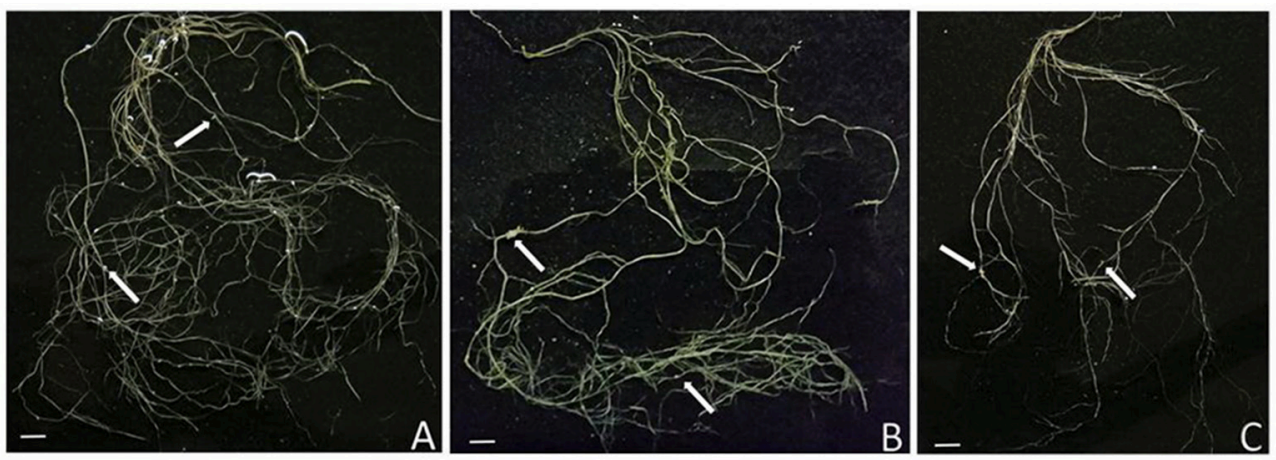

FIGURE 5 | Images are of the root system of 42-days-old $\boldsymbol{M}$. truncatula plants inoculated with $\mathbf{S}$. meliloti then grown for $\mathbf{2 8}$ dpi. The arrows indicate the position of a nodule. Note the differences in the total root length and size of nodules in each treatment. (A) Roots of vertical gravitational control seedlings. (B) Roots of horizontal control seedlings. (C) Roots from clinorotated seedlings. Scale bars $=1 \mathrm{~cm}$.

with rhizobia only, and $47.5 \pm 6.7(N=10)$ for those inoculated with both microbes. Finally, for clinorotated plants, the mean number \pm SE of nodules for plants inoculated with rhizobia only was $53.5 \pm 5.6(N=15)$ while those plants inoculated with both microbes averaged $73.9 \pm 4.15(N=15)$ nodules.

There was a significant correlation $(r=0.35, p<0.05)$ between nodule size and biomass in plants that are only inoculated with rhizobia (Figure 9A). As nodules increase in size, above-ground biomass increases as well. However, this trend was no longer significant when AMF was added to rhizobia inoculated plants (Figure 9B), suggesting AMF may indirectly influence nodule size.

\section{DISCUSSION}

The first goal of this research was to determine if the development of $M$. truncatula is changed under clinorotation. The second objective of the research was to determine if the symbiotic relationships among the host plant, rhizobia, and AMF were altered due to clinorotation. Parameters used to measure changes in $M$. truncatula development and the effects of symbioses were dry mass of aerial tissue, change in fresh mass of the host plant, total root length, and root:shoot fresh mass ratio. To determine changes between the host plant and microsymbionts under clinorotation, we measured nodule size, nodule density, and AMF percent colonization.

\section{Effects of Clinorotation on M. truncatula Growth}

It is well known that microgravity can have profound effects on the growth and development of plants. For example, the growth rate and biomass of various plant organs changes significantly in microgravity compared to $1 \mathrm{~g}$ controls (Hilaire et al., 1996; Musgrave et al., 2000; Wolverton and Kiss, 2009; Paul et al., 2012). Physiological processes such as cellular division and other parameters are altered under microgravity (Matia et al., 2010). Previous research has shown that simulated microgravity affects auxin regulation (Oka et al., 1995), which has also been shown

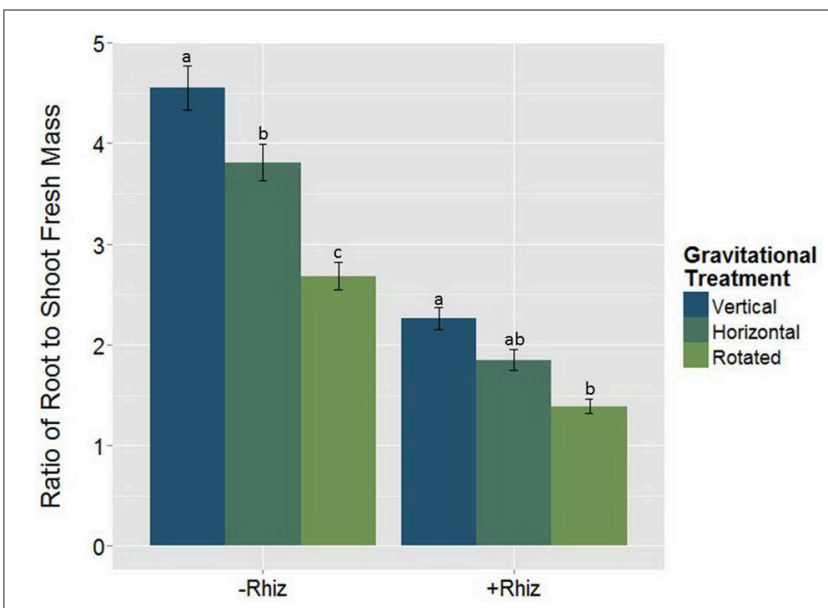

FIGURE 6 | The root:shoot fresh mass ratio responding to the significant rhizobia $\times$ gravitational treatment interaction $(p<0.05)$. The root:shoot fresh mass reduces with clinorotation and the addition of rhizobia (+Rhizobia). Rhizobia + clinorotated treatments $(n=30)$ have a significantly smaller root to shoot mass ratio than vertical controls $(p<0.05 ; n=39)$. Plants without nodules (-Rhizobia; $n=117$ ) have a higher root:shoot fresh mass ratio than + Rhizobia $(p<0.05 ; n=69)$. The letters above each standard error bar denote significance between rhizobia treatments.

to be involved in nodule development in Medicago (Wasson et al., 2006). In Arabidopsis seedlings grown on the ISS, these latter researchers found that cortical meristematic tissue had increased individual cell numbers, but growth decreased in those cells compared to $1 \mathrm{~g}$ controls. The changes in cellular processes due to true microgravity led to increased hypocotyl and total root length. In a different spaceflight experiment, seedlings of mung bean, oat, and sunflower were exposed to microgravity (Krikorian and O'Connor, 1984). In this study, the root cells showed a decreased number of cells dividing in the meristem, chromosome breakage, deletions, and translocations compared to ground controls.

The results of our research indicate that clinorotation had significant effects on $M$. truncatula compared to vertical 


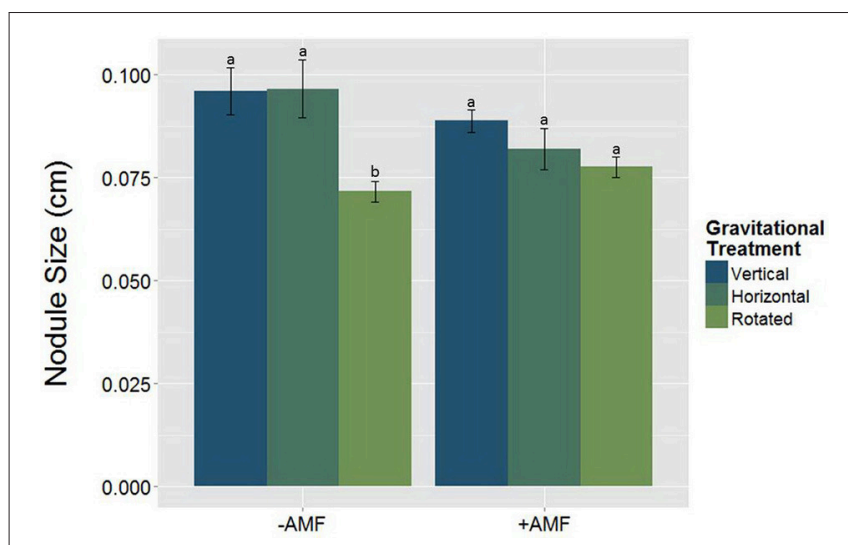

FIGURE 7 | The interaction between AMF x gravitational treatment in nodulated plants $(\boldsymbol{n}=\mathbf{7 1})$. Nodule size is significantly smaller $(\boldsymbol{p}<0.05)$ under rhizobia + clinorotated $(n=15)$ treatments compared to the static controls. The letters above each standard error bar denote significance between AMF treatments.

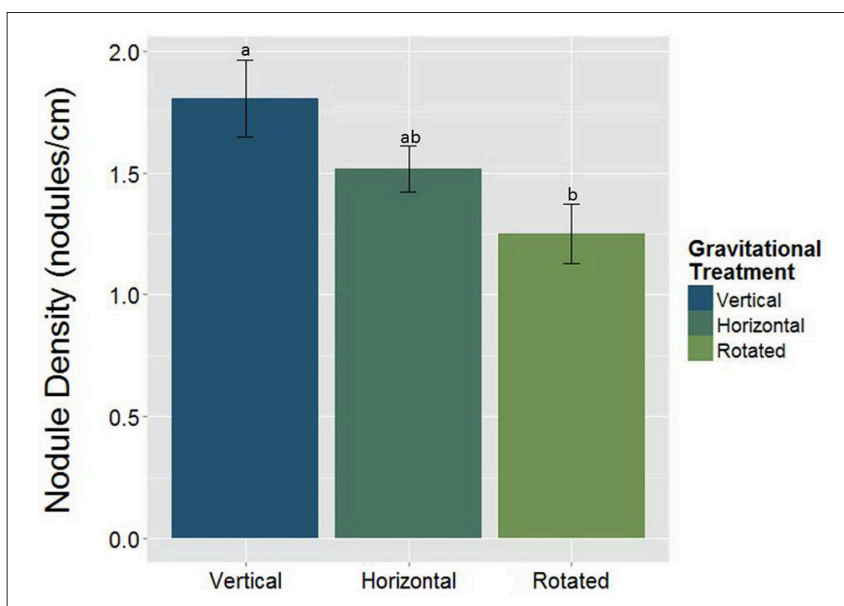

FIGURE 8 | The main effect of gravitational treatments on nodule density (nodules/cm) in $\boldsymbol{M}$. truncatula $(\boldsymbol{n}=\mathbf{7 1})$. Nodule density was calculated to compensate for the differences in total root length among gravitational treatments. The number of nodules per centimeter of root is significantly reduced in nodulated, clinorotated $(n=30)$ groups compared to the nodulated, vertical controls ( $p<0.05 ; n=19)$. The letters above each standard error bar denote significance between gravity treatments.

and horizontal growth within the microbial control group. Clinorotated plants had accumulated less total fresh and aboveground dry mass compared to the static controls (Figures 2, 3C). Similarly, Tripathy et al. (1996) found a reduction in fresh biomass of Tritium aestivium in a space experiment. Hilaire et al. (1996) also found that clinorotation reduces the fresh mass in plants compared to vertically grown plants, and attributed this result to increased ethylene production found in clinorotated plants.

In our study, total root length was reduced in clinorotated treatments. Published reports have shown an increase, decrease, or no effect (Johnson et al., 2015) in total root length after simulated or actual microgravity treatment. For instance, Levine
TABLE 6 | Two-way ANOVA results of nodule density in rhizobia and $A M F+$ rhizobia treated plants under gravitational treatments (GrT).

\begin{tabular}{lccc}
\hline \multicolumn{4}{l}{ Two-way ANOVA results of nodule density } \\
\hline Treatment & $\boldsymbol{d} \boldsymbol{f}$ & $\boldsymbol{F}$-value & $\boldsymbol{p}$-value \\
\hline $\mathrm{AMF}$ & 1 & 1.55 & 0.217 \\
${ }^{*} \mathrm{GrT}$ & 2 & 6.44 & $2.8 \times 10^{-3}$ \\
$\mathrm{AMF} \times \mathrm{GrT}$ & 2 & 4.48 & 0.159 \\
\hline
\end{tabular}

Significant differences are indicated by an asterisk $(p<0.05)$.

and Piastuch (2005) studied soybean seedlings (Glycine max) under clinorotation and found that roots were shorter in the soybean plants clinorotated compared to $1 \mathrm{~g}$ ground controls and plants grown in microgravity on the ISS. Other clinostat and spaceflight studies have shown the opposite effect (Hilaire et al., 1996; Aarrouf et al., 1999; Mortley et al., 2008). Thus, in contrast, these researchers found that in reduced gravity, plants will grow faster and are elongated compared to $1 \mathrm{~g}$ controls. Aarrouf et al. (1999) germinated plants on nutrient rich agar plates while rotating at 1 RPM on a clinostat. In contrast, after 5 days, these researchers found that horizontally clinorotated plants had increased total root length and increased fresh biomass similar to spaceflight seedlings in other experiments.

These experiments implemented by other researchers were performed on seedlings that germinated under altered gravity and for shorter periods of time in comparison to our research. Thus, young seedlings are using resources initially from cotyledons then from nutrient rich agar. Hilaire et al. (1996) grew plants in Biological Research in Canisters (BRIC), which is a sealed system that may have increased ethylene content in the surrounding environment. The host plants in this study were 14-days-old when inoculated and grown in an open environment in which ethylene could be released. Thus, these differences among experimental conditions potentially can be attributed to the deviation from morphological obtained from other studies utilizing clinorotation and space flight.

In addition, in our studies, plants grown in a horizontal orientation experienced a reduction in biomass when compared to the vertical control. We hypothesize that this slight reduction in overall plant biomass was the result of reduced physical capacity for proper root growth of the plants. As opposed to the clinorotated samples, horizontal plants had a constant gravity vector, resulting in roots growing a short distance before being impeded by the vessel wall, restricting the ability of the roots to grow and proliferate evenly throughout the soil. Due to the changing effective gravity vector of clinorotation, this same impediment was not present in clinorotated samples.

\section{The Effects of Clinorotation on M. truncatula-S. meliloti}

Our results show that over the course of these experiments, nodulated plants accumulated less fresh mass compared to the microbial control and AMF groups. Rhizobia require photosynthate from the host to fix nitrogen. Conditions and species compatibility may not have been conducive to a beneficial 

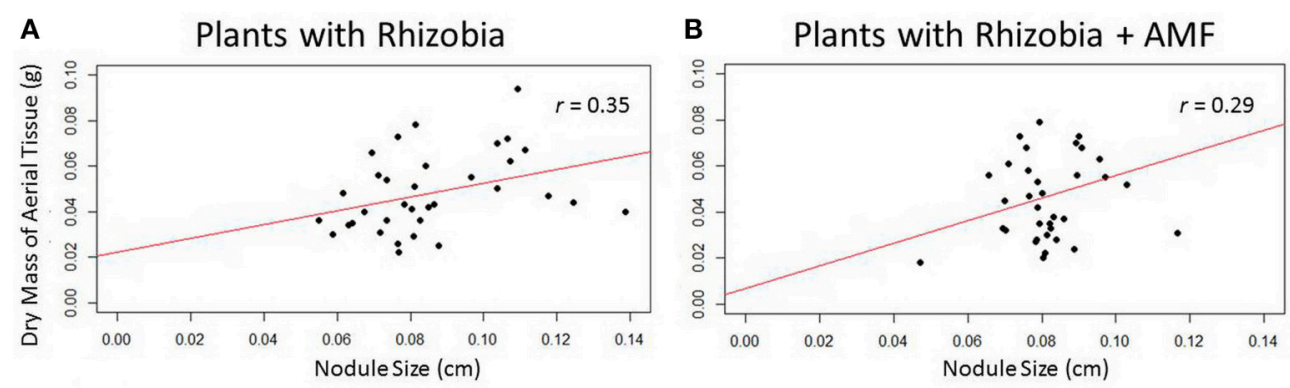

FIGURE 9 | Scatterplots depict the relationship between nodule size and dry mass of aerial tissue. (A) Plants inoculated with only rhizobia ( $n=34$ ) showed a significant $(p<0.05, p=0.35)$ positive correlation between nodule size and biomass. (B) Rhizobia + AMF $(n=35)$ inoculated plants do not have a significant correlation between nodule size and biomass.

legume-rhizobium symbiosis (Heath and Tiffin, 2007; Heath et al., 2010).

In a report by another group, Mhadhbi et al. (2005) used above-ground biomass as an indicator of nitrogenfixing efficiency in the M. truncatula-S. meliloti relationship. Their study showed that above-ground biomass was positively correlated to acetylene reduction activity, an indicator of nitrogenase activity. In our study, Figure 9A depicts the positive correlation $(r=0.35)$ between the nodule size and dry mass of aerial tissue. Plants treated with clinorotation + rhizobia have reduced nodule size causing significantly less dry aboveground biomass to accumulate. This negative trend is attenuated in clinorotation + rhizobia + AMF as the correlation is not significant between nodule size and dry above-ground mass. Furthermore, the correlation between biomass and nodule size could be due to the stress effect that clinorotation has on the symbiotic relationship, which potentially may include changes in water availability and vibrations among other things. Additional stresses such as drought and salinity stress have been shown to reduce the nitrogen-fixing ability of colonized nodules (Serraj and Drevon, 1998; Serraj et al., 1999). Previous studies on hostpathogen relationships and animal-bacteria symbiosis under altered gravity indicate that colonization of the host by the microbe is significantly altered (Bishop et al., 1997; Ryba-White et al., 2001; McFall-Ngai et al., 2010). Thus, the stress of altered gravity may inhibit rhizobia benefits by reducing the efficiency of nitrogen fixation.

\section{Clinorotation Influences Relationships among Host and Microbes}

In previous studies, host-microbe interactions are known to be affected in various ways by microgravity. Ryba-White et al. (2001) studied changes in virulence of the soybean fungal pathogen, Phytophthora sojea in a spaceflight experiment. Plants inoculated with the fungus had significantly more colonized roots while exposed to microgravity compared to ground controls. The researchers attributed the increased virulence of the pathogen to the stress of microgravity weakening the plants immune response. Similarly, Bishop et al. (1997) sent seeds of wheat cv. Super Dwarf to the ISS for a study on peroxidase activity but found that a fungal pathogen, Neotyphodium sp., had infected the germplasm of the seeds. Once germination took place, the fungus began to infect $50 \%$ of the plants grown in space while only $30 \%$ were affected in the ground controls.

Another mutualistic symbiosis studied in reduced gravity was the water fern Azolla and nitrogen-fixing cyanobacteria, Anabeana azollea, along with the concomitant bacteria associated with the symbiotic partners (Kordyum et al., 1983). In this study, the Azolla-Anabeana were sent into space for 8 days along with concomitant bacteria to test the effects of microgravity on the microcosm within the Azolla system. The cyanobacteria, Anabeana, was reported to have increased in number in the space flight experiment along with the concomitant bacteria living within the Azolla, although only data from the unknown bacteria were present in the paper. Currently, parasitic relationships among plants and microbes under microgravity are better understood than the mutualistic relationships, which are the focus of the present study.

\section{Effects of Both AMF and Rhizobia on $M$. truncatula under Clinorotation}

AMF and rhizobia often cohabitate the same host roots. Larimer and Bever (2014) used the perennial prairie legume, Amorpha canescens, to study the effects of the symbionts on the host. Their studies showed that in this legume, AMF-rhizobia symbionts benefit the plant as well as each other. The AMF-rhizobia symbionts increased above-ground biomass and provided more nitrogen and phosphorus to the above- and below-ground tissue compared to plants colonized by only one of the symbionts. AMF-rhizobia compatibility dictates the extent of nutritional benefits received by the host plant (Lisette et al., 2003). In plants inoculated with compatible AMF and rhizobia, nodule count and mass increased leading to higher nitrogen and phosphorus in shoots, above-ground biomass and increased fruit output (Lisette et al., 2003; Larimer and Bever, 2014). Thus, by inoculating legumes with two synergistic microbial symbionts, the host can provide more resources that are essential to humans on Earth (or in space).

Under $1 \mathrm{~g}$ conditions, AMF in combination with rhizobia have beneficial effects on plant growth and health (Vazquez et al., 2001; Gao et al., 2012; Wang et al., 2012). Our findings show that nodule density is reduced in rhizobia + clinorotated 
treated plants compared to vertical gravitational control. In our studies, although the rhizobia + horizontal control is not significantly different from the clinorotated group, there is a substantial increase in nodules/cm within the horizontal group in comparison. Heath et al. (2010) describe nodule numbers as the trait most likely to be "the main determinant of rhizobium fitness during the symbiotic stage of the life cycle." The addition of AMF and clinorotation may reduce the ability of rhizobia to form nodules on the roots of a host within the time parameters of our experiment. Studies by other researchers show that under the growth conditions found on Earth, AMF can inhibit rhizobia species from nodulation when co-inoculated (Lisette et al., 2003; Mortimer et al., 2008; Wang and Dong, 2011).

Interestingly, it appears that rhizobia inoculation tends to have an overall negative effect on plant biomass accumulation, while AMF inoculation tends to have a more beneficial effect. In treatments where both microbes were present, the rhizobia effect of reduced biomass accumulation by the plant is still present. As part of the symbiotic relationship between rhizobia and $M$. truncatula, carbohydrates are provided to the bacteria in exchange for nitrogen. It is likely that the cost of this mutualistic relationship reduces the amount of carbohydrates available for overall biomass production of the plant.

In summary, our results clearly demonstrate that clinorotation reduces the growth and development of $M$. truncatula and

\section{REFERENCES}

Aarrouf, J., Darbelley, N., Demandre, C., Razafindramboa, N., and Perbal, G. (1999). Effect of horizontal clinorotation on the root system development and on lipid breakdown in rapeseed (Brassica napus) seedlings. Plant Cell Physiol. 40, 396-405. doi: 10.1093/oxfordjournals.pcp.a0 29555

Ashrafi, E., Zahedi, M., and Razmjoo, J. (2014). Co-inoculations of arbuscular mycorrhizal fungi and rhizobia under salinity in alfalfa. Soil Sci. Plant Nutr. 60, 619-629. doi: 10.1080/00380768.2014.936037

Bécard, G., and Fortin, J. A. (1988). Early events of vesicular-arbuscular mycorrhiza formation on Ri-T-DNA transformed roots. New Phytol. 108, 211-218. doi: 10.1111/j.1469-8137.1988.tb03698.x

Bishop, D. L., Levine, H. G., Kropp, B. R., and Anderson, A. J. (1997). Seedborne fungal contamination: consequences in space-grown wheat. Phytopathology 87, 1125-1133. doi: 10.1094/PHYTO.1997.87.11.1125

Colla, G., Battistelli, A., Proietti, S., Moscatello, S., Rouphael, Y., Cardarelli, M., et al. (2007). Rocket seedling production on the International Space Station: growth and nutritional properties. Microgravity Sci. Tech. 19, 118-121. doi: 10.1007/BF02919465

Connor, D. J., Loomis, R. S., and Cassman, K. G. (2011). Crop Ecology: Productivity and Management in Agricultural Systems. New York, NY: Cambridge University Press.

Ferl, R., Wheeler, R., Levine, H. G., and Paul, A. L. (2002). Plants in Space. Curr. Opin. Plant Bio. 5, 258-263. doi: 10.1016/S1369-5266(02)00254-6

Galkovskyi, T., Mileyko, Y., Bucksch, A., Moore, B., Symonova, O., Price, C. A., et al. (2012). GiA Roots: software for the high-throughput analysis of plant root system architecture. BMC Plant Biol. 12:116. doi: 10.1186/1471-2229-12-116

Gao, X., Lu, X., Wu, M., Zhang, H., Pan, R., Tian, J., et al. (2012). Co-inoculation with rhizobia and AMF inhibited soybean red crown rot: from field study to plant defense - related gene expression analysis. PLoS ONE 7:e33977. doi: 10.1371/journal.pone.0033977

Graham, P. H., and Vance, C. P. (2003). Legumes: importance and constraints to greater use. Plant Physiol. 131, 872-877. doi: 10.1104/pp.017004 significantly affects the tripartite symbiosis among $M$. truncatula, $S$. meliloti, and R. irregularis. Nodulation is affected in both groups of microbial and clinorotated treatments and the most notable effect of clinorotation is the reduction in nodule size and number. AMF colonization was not significantly altered by clinorotation in these studies, but further research into the relationship of the compatibility of the plant-microbe relationship under altered gravity is needed. In the long-term, these results on the effects of clinorotation on symbiosis need to be extended in spaceflight experiments. Furthermore, careful consideration of the symbiotic microbes of plants needs to be included in the design of self-sustaining life support systems needed for space travel.

\section{AUTHOR CONTRIBUTIONS}

$\mathrm{AD}$ designed and performed the experiments, analyzed the results, and wrote the manuscript. JV participated in the analysis of the results and the writing of the manuscript. JK supervised the project, and participated in the analysis and writing of the manuscript.

\section{FUNDING}

Financial support for this project was provided by NASA grant NNX15AK39A.
Heath, K. D., Stock, A. J., and Stinchcombe, J. R. (2010). Mutualism variation in the nodulation response to nitrate. J. Evol. Biol. 23, 2494-2500. doi: 10.1111/j.14209101.2010.02092.x

Heath, K. D., and Tiffin, P. (2007). Context dependence in coevolution of plant and rhizobial mutualists. Proc. Biol. Sci. 274, 1905-1912. doi: 10.1098/rspb.2007.0495

Herranz, R., Anken, R., Boonstra, J., Braun, M., Christianen, P. C. M., de Geest, M., et al. (2013). Ground-based facilities for simulation of microgravity: organismspecific recommendations for their use, and recommended terminology. Astrobiology 13, 1-17. doi: 10.1089/ast.2012.0876

Hilaire, E. M., Peterson, B. V., Guikema, J. A., and Brown, C. S. (1996). Clinorotation affects morphology and ethylene production in soybean seedlings. Plant Cell Physiol. 37, 929-934. doi: 10.1093/oxfordjournals.pcp.a029041

Hogekamp, C., and Kuster, H. (2013). A roadmap of cell-type specific gene expression during sequential stages of arbuscular mycorrhiza symbiosis. BMC Genomics 14:306. doi: 10.1186/1471-2164-14-306

Johnson, C. M., Subramaniana, A., Edelmann, R. E., and Kiss, J. Z. (2015). Morphometric analyses of petioles of seedlings grown in a spaceflight experiment, J. Plant Res. 128, 1007-1016. doi: 10.1007/s10265-015-0749-0

Journet, E. P., de Carvalho-Niebel, F., Andriankaja, A., Huguet, T., and Barker, D. G. (2006). "Rhizobial inoculation and nodulation of Medicago truncatula," in The Medicago truncatula Handbook, eds U. Mathesius, E. P. Journet, and L. W. Sumner. Available online at: http://www.noble.org/MedicagoHandbook/

Kiss, J. Z. (2000). Mechanisms of the early phases of plant gravitropism. Crit. Rev. Plant Sci. 19, 551-573. doi: 10.1016/S0735-2689(01)80008-3

Kordyum, V. A., Man'ko, V. G., Popve, A. F., Mashinsky, A. L., Shcherbak, O. H., and Thoyk, N. G. (1983). Changes in symbiotic and associative interrelations in a higher plant-bacterial system during space flight. Adv. Space Res. 3, 265-268.

Kouchi, H., Imaizumi-Anraku, H., Hayashi, M., Hakoyama, T., Nakagawa, T., Umehara, Y., et al. (2010). How many peas in a pod? Legume genes responsible for mutualistic symbioses underground. Plant Cell Physiol. 51, 1381-1397. doi: $10.1093 / \mathrm{pcp} / \mathrm{pcq} 107$ 
Kraft, T. F., van Loon, J. J., and Kiss, J. Z. (2000). Plastid position in Arabidopsis columella cells is similar in microgravity and on a random-positioning machine. Planta 211, 415-422. doi: 10.1007/s004250000302

Krikorian, A. D., and O'Connor, S. A. (1984). Karyological observations. Ann. Bot. 54, 49-63.

Larimer, A. L., and Bever, J. D. (2014). Synergism and context dependency of interactions between arbuscular mycorrhizal fungi and rhizobia with a prairie legume. Ecology 95, 1045-1054. doi: 10.1890/13-0025.1

Larimer, A. L., Bever, J. D., and Clay, K. (2010). The interactive effects of plant microbial symbionts a review and meta-analysis. Symbiosis 51, 139-148. doi: 10.1007/s13199-010-0083-1

Levine, H. G., and Piastuch, W. C. (2005). Growth patterns for etiolated soybeans germinated under spaceflight conditions. Adv. Space Res. 36, 1237-1243. doi: 10.1016/j.asr.2005.02.050

Lisette, J., Xavier, C., and Germida, J. J. (2003). Selective interactions between arbuscular mycorrhizal fungi and Rhizobium leguminosarum vc Viceae enhance pea yield and nutrition. Biol. Fertil. Soil 37, 261-267. doi: 10.1007/s00374-0030605-6

Matia, I., Gonzalez-Camacho, F., Herranz, R., Kiss, J. Z., Gasset, G., van Loon, J. J., et al. (2010). Plant cell proliferation and growth are altered by microgravity conditions in spaceflight. J. Plant Physiol. 167, 184-193. doi: 10.1016/j.jplph.2009.08.012

McFall-Ngai, M. J., Nyholm, S. V., and Castillo, M. G. (2010). The role of the immune system in the initiation and persistence of the Euprymna scolopes-Vibrio fischeri symbiosis. Semin. Immunol. 22, 48-53. doi: 10.1016/j.smim.2009.11.003

Mhadhbi, H., Jebara, M., Limam, F., Huguet, T., and Aouani, M. E. (2005). Interaction between Medicago truncatula lines and Sinorhizobium meliloti strains for symbiotic efficiency and nodule antioxidant activites. Physiol. Plant. 124, 4-11. doi: 10.1111/j.1399-3054.2005.00489.x

Mortimer, P. E., Perez-Fernandez, M. A., and Valentine, A. J. (2008). The role of arbuscular mycorrhizal colonization in the carbon and nutrient economy of the tripartite symbiosis with nodulated Phaseolus vularis. Soil Biol. Biochem. 40, 1019-1027 doi: 10.1016/j.soilbio.2007.11.014

Mortley, D. G., Bonsi, C. K., Hill, W. A., and Morris, C. A. (2008). Influence of microgravity environment on root growth, soluble sugars, and starch concentration of sweetpotato stem cuttings. J. Am. Soc. Hortic. Sci. 133, $327-332$.

Musgrave, M. E., Kuang, A., Xiao, Y., Stout, S. C., Bingham, G. E., Briarty, L. G., et al. (2000). Gravity independence in seed-to seed cycling in Brassica rapa. Planta 210, 400-406. doi: 10.1007/PL00008148

Nadeem, S. M., Ahmad, M., Zahir, Z. A., Javaid, A., and Ashraf, M. (2014). The role of mycorrhizae and plant growth promoting rhizobacteria (PGPR) in improving crop productivity under stressful environments. Biotechnol. Adv. 32, 429-448. doi: 10.1016/j.biotechadv.2013.12.005

National Research Council (2011). Recapturing a Future for Space Exploration: Life and Physical Sciences Research for a New Era. Washington, DC: The National Academies Press.

Oka, M., Ueda, J., Miyamoto, K., Yamamoto, R., Hoson, T., and Kamisaka, S. (1995). Effect of simulated microgravity on auxin polar transport in inflorescence axis in Arabidopsis thaliana. Biol. Sci. Space 9, 331-336. doi: $10.2187 /$ bss. 9.331
Paul, A. L., Amalfitano, C. E., and Ferl, R. J. (2012). Plant growth strategies are remodeled by spaceflight. BMC Plant Bio. 12:232. doi: 10.1186/1471-222912-232

Qian, A. R., Yin, D. C., Yang, P. F., Lv, Y., Tian, Z. C., and Shang, P. (2013). Application of diamagnetic levitation technology in biological science research. IEEE Trans. Appl. Supercond. 23, 1-5. doi: 10.1109/TASC.2012.2232919

Rasband, W. S. (2014). ImageJ, U. S. National Institutes of Health. Bethesda, MD. Available online at: http://imagej.nih.gov/ij/

Ryba-White, M., Nedukha, O., Hilaire, E., Guikema, J. A., Kordyum, E., and Leach, J. E. (2001). Growth in microgravity increases susceptibility of soybean to a fungal pathogen. Plant Cell Physiol. 42, 657-664. doi: 10.1093/pcp/ pce082

Serraj, R., and Drevon, J. J. (1998). Effects of salinity and nitrogen source growth and nitrogen fixation on alfalfa. J. Plant Nutr. 21, 1805-1818. doi: 10.1080/01904169809365525

Serraj, R., Sinclair, T. R., and Purcell, L. C. (1999). Symbiotic N2 fixation response to drought. J. Exp. Bot. 50, 143-155. doi: 10.1093/jxb/50.331.143

Smith, S. E., and Read, D. J. (2008). Mycorrhizal Symbiosis, 3rd Edn. San Diego, CA: Academic Press.

Tripathy, B. C., Christopher, S. B., Levine, H. G., and Krikorian, A. D. (1996). Growth and photosynthetic response of wheat plants grown in space. Plant Physiol. 110, 801-806. doi: 10.1104/pp.110.3.801

Vandenbrink, J. P., and Kiss, J. Z. (2016). Space, the final frontier: A critical review of recent experiments performed in microgravity. Plant Sci. 243, 115-119. doi: 10.1016/j.plantsci.2015.11.004

Vazquez, M. M., Azcon, R., and Barea, J. M. (2001). Compatibility of a wild type and its genetically modified Sinorhizobium strain with two mycorrhizal fungi on Medicago species as affected by drought stress. Plant Sci. 161, 347-348. doi: 10.1016/S0168-9452(01)00416-2

Wang, D., and Dong, X. (2011). A highway for war and peace: the secretory pathway in plant-microbe interactions. Mol. Plant 4, 581-587. doi: 10.1093/ $\mathrm{mp} / \mathrm{ssr} 053$

Wang, D., Yang, S., Tang, F., and Zhu, H. (2012). Symbiosis specificity in the legume - rhizobial mutualism. Cell. Microbiol. 14, 334-342. doi: 10.1111/j.14625822.2011.01736.x

Wasson, A. P., Pellerone, F. I., and Mathesius, U. (2006). Silencing the flavonoid pathway in Medicago truncatula inhibits root nodule formation and prevents auxin transport regulation in Rhizobia. Plant Cell 18, 1617-1629. doi: $10.1105 /$ tpc. 105.038232

Wolverton, S. C., and Kiss, J. Z. (2009). An update on plant science biology. Gravit. Space Biol. Bull. 22, 13-20.

Conflict of Interest Statement: The authors declare that the research was conducted in the absence of any commercial or financial relationships that could be construed as a potential conflict of interest.

Copyright (c) 2016 Dauzart, Vandenbrink and Kiss. This is an open-access article distributed under the terms of the Creative Commons Attribution License (CC BY). The use, distribution or reproduction in other forums is permitted, provided the original author(s) or licensor are credited and that the original publication in this journal is cited, in accordance with accepted academic practice. No use, distribution or reproduction is permitted which does not comply with these terms. 